\title{
On-chip nano-electro-mechanical switching of deterministic single photons
}

Zhou, Xiaoyan; Papon, Camille; Thyrrestrup, Henri; Liu, Zhe; Stobbe, Søren; Schott, Rudiger; Wieck, Andreas D.; Ludwig, Arne; Lodahl, Peter; Midolo, Leonardo

\section{Published in:}

Proceedings of 2019 Conference on Lasers and Electro-Optics

Link to article, DOI:

10.23919/CLEO.2019.8749753

Publication date:

2019

Document Version

Peer reviewed version

Link back to DTU Orbit

Citation (APA):

Zhou, X., Papon, C., Thyrrestrup, H., Liu, Z., Stobbe, S., Schott, R., Wieck, A. D., Ludwig, A., Lodahl, P., \& Midolo, L. (2019). On-chip nano-electro-mechanical switching of deterministic single photons. In Proceedings of 2019 Conference on Lasers and Electro-Optics [8749753] IEEE. 2019 Conference on Lasers and ElectroOptics, CLEO 2019 - Proceedings https://doi.org/10.23919/CLEO.2019.8749753

\section{General rights}

Copyright and moral rights for the publications made accessible in the public portal are retained by the authors and/or other copyright owners and it is a condition of accessing publications that users recognise and abide by the legal requirements associated with these rights.

- Users may download and print one copy of any publication from the public portal for the purpose of private study or research.

- You may not further distribute the material or use it for any profit-making activity or commercial gain

- You may freely distribute the URL identifying the publication in the public portal 


\title{
On-chip nano-electro-mechanical switching of deterministic single photons
}

\author{
Xiaoyan Zhou ${ }^{1}$, Camille Papon ${ }^{1}$, Henri Thyrrestrup ${ }^{1}$, Zhe Liu $^{1}$, Søren Stobbe ${ }^{2, *}$, \\ Rüdiger Schott ${ }^{3}$, Andreas D. Wieck ${ }^{3}$, Arne Ludwig ${ }^{3}$, Peter Lodahl ${ }^{1}$, and Leonardo \\ Midolo ${ }^{1}$ \\ ${ }^{1}$ Center for Hybrid Quantum Networks (Hy-Q), Niels Bohr Institute, University of Copenhagen, Blegdamsvej 17, \\ 2100-DK Copenhagen, Denmark \\ ${ }^{2}$ Niels Bohr Institute, University of Copenhagen, Blegdamsvej 17, 2100-DK Copenhagen, Denmark \\ ${ }^{3}$ Lehrstuhl für Angewandte Festkörperphysik, Ruhr-Universität Bochum, Universitätsstrasse 150, D-44780 \\ Bochum, Germany \\ * Present affiliation: Department of Photonics Engineering, DTU Fotonik, Technical University of Denmark, \\ Building 343, 2800 Kongens Lyngby, Denmark \\ xiaoyan.zhou@nbi.ku.dk
}

\begin{abstract}
We demonstrate a nano-electro-mechanical single-photon router integrated with semiconductor quantum emitters, showing an extinction ratio of $>20 \mathrm{~dB}$ and operation speed of $\mathrm{MHz}$ with insertion loss of $0.67 \mathrm{~dB}$ and footprint $<30 \mu \mathrm{m}^{2}$. (C) 2019 The Author(s)

OCIS codes: (270.0270) Quantum optics, (230.4685) Optical microelectromechanical devices, (130.3120) Integrated optics devices.
\end{abstract}

Quantum photonic integrated circuits enable scaling up quantum optics experiments by integrating multiple functionalities, such as deterministic photon-emitter interfaces, routers, and detectors, on a chip. To this end, a key requirement is to develop reconfigurable circuitry that can be operated at a speed compatible with the emitter qubit coherence time $(0.1-1 \mu \mathrm{s})$ and introduces neither loss nor emitter decoherence. The existing tuning mechanisms, based on thermo-optic and electro-optic effects, do not meet these demands as they are either too slow or require very large footprints, limiting both scalability and efficiency. In this work, we demonstrate a different approach to on-chip routing by using nano-electro-mechanical devices integrated with deterministic single-photon sources, i.e., InAs quantum dots (QDs) in GaAs. Unlike refractive-index-tuning methods, such electro-opto-mechanical interaction can be very strong and material-independent [1], thus offering the advantage of a much smaller attainable device footprint, lower insertion loss, faster switching speed, and better scalability.
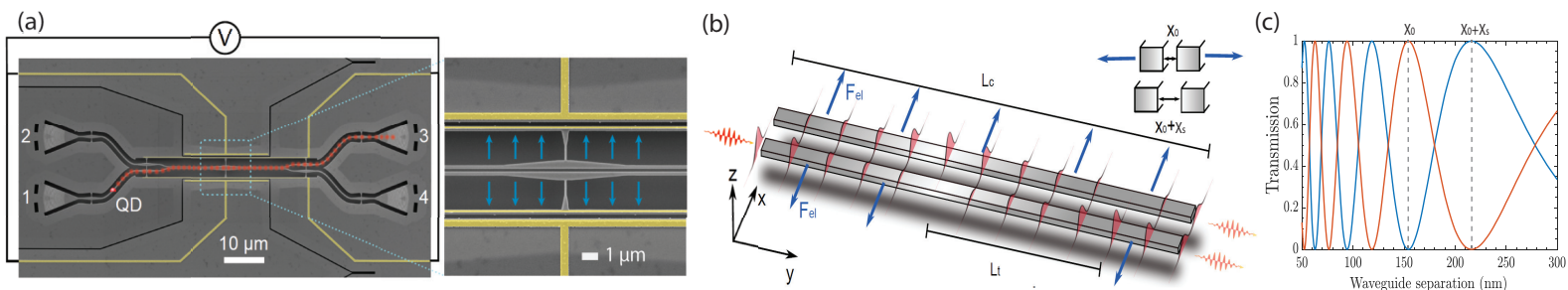

Fig. 1. (a) Scanning electron micrograph image of the nano-electro-mechanical router on GaAs membrane with embedded InAs quantum dots. (b) Schematic of the device working principle. (c) Transmission at two output ports as a function of gap distance.

The integrated photon router is fabricated in 160-nm thick GaAs membranes with embedded InAs QDs, as shown by a scanning electron micrograph in Fig. 1(a). It consists of two identical suspended nanobeam waveguides each attached to capacitive nanomechanical actuators, forming a directional coupler with controllable gap distance $d$ and, therefore, tunable coupling strength $g(d)$. In the case of illumination from one waveguide, photons are fully transferred to the other waveguide after a length $L_{t}(d)=\pi /(2 g(d))$. The splitting ratio at the end of the coupling region (length $\left.L_{c}\right)$ is $\mathrm{SR}=\tan ^{2}\left(\left(\pi L_{c}\right) /\left(2 L_{t}(d)\right)\right)$. By tuning the gap distance with electro-static force, photons can be deterministically switched between two output ports, as shown in Figs. 1(b) and 1(c). The effect of tuning is more pronounced with a smaller starting gap distance $\left(x_{0}\right)$, showing the advantage of working at the nanoscale.

We characterize our device in a Helium flow cryostat at $10 \mathrm{~K}$. Multiple QDs in one arm of the waveguide (Port 1) are optically excited and the emission spectra, collected from Port 3 and Port 4 at different driving voltages, are 
shown in Fig. 2(a). A clear anti-correlation between the two ports can be found. Unlike thermo-optical methods, the absence of de-tuning of the excitonic emission energy confirms that disturbance-free single-photon routing through electro-mechanical approach is achieved. The integrated and normalized photon counts of an emission line at a wavelength of $927.26 \mathrm{~nm}$, shown in Fig. 2(b), indicate a maximum extinction ratio of (23.4 \pm 1.7$) \mathrm{dB}$ at a bias voltage of $7 \mathrm{~V}$, corresponding to a displacement $x_{s}=24 \mathrm{~nm}$ (see ref. [2]). The single-photon nature of the routing is confirmed by a pulsed Hanbury-Brown and Twiss experiment, showing a single-photon purity $g^{(2)}(0)=$ $0.18 \pm 0.03$ (see ref. [2]).
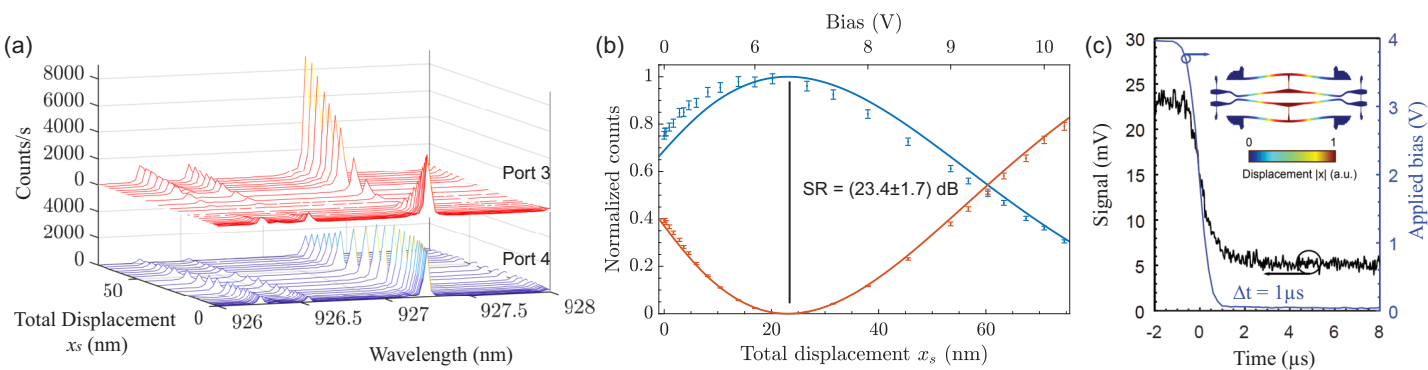

Fig. 2. Routing single photons from quantum dots: (a) emission spectra and (b) integrated and normalized transmission from a single excitonic line at $927.26 \mathrm{~nm}$ from two output ports as a function of displacement. (c) Ring-down response of the router showing operation speed in the $\mathrm{MHz}$ regime.

The time response of the router is limited by the fundamental resonant frequency $v_{m}$, which, from numerical simulation (inset of Fig. 2(c)), is estimated at around $1.36 \mathrm{MHz}$, enabling sub-microsecond switching time. To verify this, we perform a ring-down measurement by applying a voltage of $4 \mathrm{~V}$ with a ramp-down time $\Delta t=1 \mu s$. To avoid damaging the device due to excessive oscillation at resonance, the sample is tested at room temperature and at ambient pressure to dampen the mechanical quality factor. As shown in Fig. 2(c), the measured optical signal, which probes the mechanical motion of the waveguides, follows the dynamics of the input bias signal, indicating that the device can be driven at $\mathrm{MHz}$ speed. Moreover, resonant actuation schemes could be used to route photons from port to port at $1 /\left(2 v_{m}\right) \sim 370 \mathrm{~ns}$, for applications such as on-chip de-multiplexing [3].

By comparing the loss of the router to suspended waveguides, we estimate a total insertion loss of 0.67 $\mathrm{dB} / \mathrm{switch}$. The demonstrated high performance of the single-photon router opens new opportunities for building de-multiplexed sources of $N$ single photons. Given the low insertion loss, a 10-photon source can be readily achieved by cascading the nanomechanical routers. With the use of QDs at telecom wavelengths and further realistic device optimization, the technology could be scaled up to achieve $N>50$, required to show quantum advantage for boson sampling. Furthermore, adding the spin quantum memory could lead to a new generation of non-trivial photonic quantum resources.

In summary, we present a nano-electro-mechanical reconfigurable single-photon router integrated with QDs as deterministic sources. The demonstrated extinction ratio of $>20 \mathrm{~dB}$, low insertion loss of $0.67 \mathrm{~dB} / \mathrm{switch}$ and operation speed of $\mathrm{MHz}$, combined with the small footprint and the planar design, allow cascading multiple devices in a single chip, opening new opportunities for complex on-chip quantum experiments such as de-multiplexing single-photon sources and programmable gates for boson sampling and quantum simulation.

We gratefully acknowledge financial support from the Danish National Research Foundation (Center of Excellence Hy-Q), the European Research Council (ERC Advanced Grant SCALE and ERC-PoC Grant 'FIPS'), Innovation Fund Denmark (Quantum Innovation Center Qubiz), the Villum Foundation, the Danish Research Infrastructure Grant (QUANTECH), and the Danish Council for Independent Research (grant number 4184-00203). A.L., R.S., and A.D.W. acknowledge support of BMBF (Q.Link.X 16KIS0867) and the DFG (TRR 160).

\section{References}

1. Leonardo Midolo, Albert Schliesser, and Andrea Fiore. Nano-opto-electro-mechanical systems. Nature nanotechnology, 13(1):11, 2018.

2. Camille Papon, Xiaoyan Zhou, Henri Thyrrestrup, Zhe Liu, Søren Stobbe, Rüdiger Schott, Andreas D Wieck, Arne Ludwig, Peter Lodahl, and Leonardo Midolo. Nanomechanical single-photon routing. arXiv preprint arXiv:1811.10962, 2018.

3. Hui Wang, Wei Li, Xiao Jiang, Y-M He, Y-H Li, Xing Ding, M-C Chen, Jian Qin, C-Z Peng, Christian Schneider, et al. Toward scalable boson sampling with photon loss. Physical Review Letters, 120(23):230502, 2018. 